EPJ Web of Conferences 66, 05010 (2014)

DOI: $10.1051 /$ epjconf/ 20146605010

(C) Owned by the authors, published by EDP Sciences, 2014

\title{
Near-infrared laser spectroscopy of antiprotonic helium atoms
}

\author{
T. Kobayashi, ${ }^{1}$, , D. Barna ${ }^{1,2}$, R. S. Hayano ${ }^{1}$, Y. Murakami ${ }^{1}$, K. Todoroki ${ }^{1}$, H. Yamada ${ }^{1}$, A. Dax ${ }^{3}$, L. \\ Venturelli $^{4,5}$, N. Zurlo ${ }^{4,5}$, D. Horváth ${ }^{2,6}$, H. Aghai-Khozani ${ }^{7,8}$, A. Sótér ${ }^{7}$, and M. Hori ${ }^{7,1}$ \\ ${ }^{1}$ Department of Physics, University of Tokyo, Hongo, Bunkyo-ku 113-0033, Japan \\ ${ }^{2}$ Institute for Particle and Nuclear Physics, Wigner Research Centre for Physics, Hungarian Academy of \\ Sciences, P.O.Box 49, Budapest, 1525, Hungary \\ ${ }^{3}$ Paul Scherrer Institute, 5232 Villigen-PSI, Switzerland \\ ${ }^{4}$ Dipartimento di Ingegneria dell' Informazione, Universitá di Brescia, 25123 Brescia, Italy \\ 5 INFN, Gruppo Collegato di Brescia, 25133 Brescia, Italy \\ ${ }^{6}$ Institute of Nuclear Research, H-4001 Debrecen, Hungary \\ ${ }^{7}$ Max-Planck-Institut für Quantenoptik, Hans-Kopfermann-Strasse 1, D85748 Garching, Germany \\ ${ }^{8} \mathrm{CERN}, \mathrm{CH}-1211$ Geneva 23, Switzerland
}

\begin{abstract}
The ASACUSA (Atomic Spectroscopy and Collisions Using Slow Antiprotons) collaboration is currently attempting to observe a laser-induced resonant transition of antiprotonic helium atoms at wavelength of $1154.9 \mathrm{~nm}$. Motivations to observe this transition and a nanosecond near-infrared laser prepared for this work are presented.
\end{abstract}

\section{Introduction}

The antiprotonic helium atom $\overline{\mathrm{p}} \mathrm{He}^{+}$is a three-body system consisting of an antiproton $\overline{\mathrm{p}}$, an electron $\mathrm{e}^{-}$, and a helium nucleus $\mathrm{He}^{2+}[1,2]$. The $\overline{\mathrm{p}} \mathrm{He}^{+}$atoms with antiprotons populating Rydberg states having large principal $(n \sim 38)$ and angular momentum $(l \sim n-1)$ quantum numbers are metastable (i.e., the atoms have microsecond-scale lifetimes against antiproton annihilations). Laser spectroscopy of the $\overline{\mathrm{p}} \mathrm{He}^{+}$atoms has been carried out by the ASACUSA collaboration at the Antiproton Decelerator (AD) of CERN [3-7]. By comparing the measured transition frequencies of the $\overline{\mathrm{p}} \mathrm{He}^{+}$atoms with three-body QED calculations [8], the antiproton-to-electron mass ratio was determined as $M_{\overline{\mathrm{p}}} / m_{\mathrm{e}}=$ 1836.1526736(23) [7].

The ASACUSA collaboration is currently attempting to observe the transition $(n, l)=(40,36) \rightarrow$ $(41,35)$ of the $\overline{\mathrm{p}}^{4} \mathrm{He}^{+}$isotope at wavelength $\lambda=1154.9 \mathrm{~nm}$ (see figure 1 ). This wavelength is longer than that of any transition observed in the $\overline{\mathrm{p}} \mathrm{He}^{+}$atoms so far. The method of the laser spectroscopy is similar to that of Ref. [3]. A pulsed antiproton beam with a momentum of $100 \mathrm{MeV} / c$ is extracted from the $\mathrm{AD}$. The $\overline{\mathrm{p}} \mathrm{He}^{+}$atoms are produced by stopping the antiprotons in a cryogenic helium gas target [9]. A laser induces the transition between the metastable state $(40,36)$ and the short-lived state $(41,35)$ with nanosecond-scale lifetime against Auger emission of the electron (see Sect. 2). A $\bar{p} \mathrm{He}^{2+}$ ion, which remains after the Auger emission, is rapidly destroyed by collisions with the surrounding helium atoms. Charged pions emerging from antiproton annihilations are detected by a Cherenkov counter [10].

\footnotetext{
ae-mail: tkobayashi@nucl.phys.s.u-tokyo.ac.jp
} 


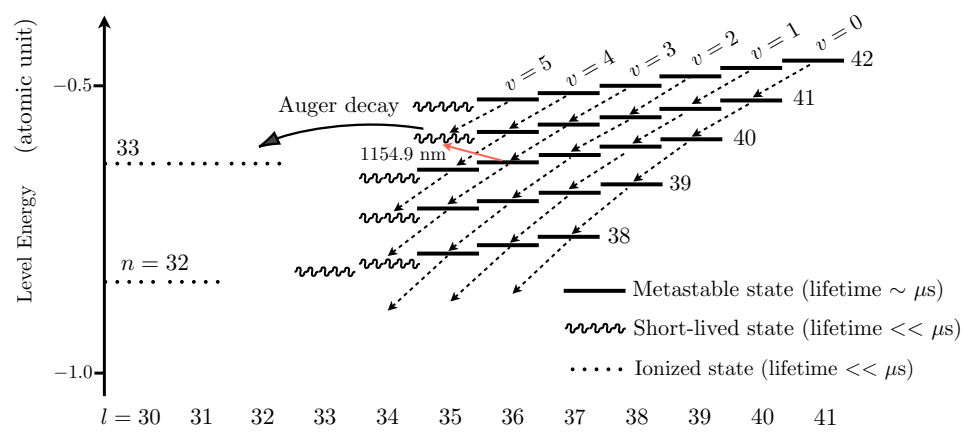

Figure 1. Energy level diagram of the $\overline{\mathrm{p}}^{4} \mathrm{He}^{+}$isotope. The $1154.9 \mathrm{~nm}$ transition is indicated with a red arrow. Dashed arrows indicate radiative decays with microsecond-scale lifetimes. The ionized $l$-states with the same $n$ are denerate.

\section{Motivations to observe the $1154.9 \mathrm{~nm}$ transition}

\subsection{Three-body calculation of the Auger decay rate}

Some of the Rydberg $\overline{\mathrm{p}} \mathrm{He}^{+}$states have short lifetimes against Auger emission of the $1 S$ electron,

$$
\left(\overline{\mathrm{p}} \mathrm{He}^{+}\right)_{n, l} \rightarrow\left(\overline{\mathrm{p}} \mathrm{He}^{2+}\right)_{n^{\prime}, l^{\prime}}+\mathrm{e}^{-} .
$$

This Auger decay process is strongly suppressed for high transition multipolarity $L=l-l$, i.e., the angular momentum transfer from the antiproton to the electron. The Auger decay rate $\gamma_{\mathrm{A}}$ can be approximately estimated as $[1,2]$,

$$
\gamma_{\mathrm{A}} \sim 10^{17-3 L} \mathrm{~s}^{-1} .
$$

Eq. (2) indicates that only states with $L \leq 3$ are Auger-dominated, i.e., they have fast decay rates compared to those of the metastable states.

We plan to deduce the decay rate of the state $(41,35)$ with the lowest possible $L=3$ (see figure 1). This value may be compared with a theoretical Auger decay rate obtained by a variational three-body calculation using the complex-coordinate rotation method [8]. In general, accurate calculations of the Auger decay rates are difficult since a finite set of variational wavefunctions are used to represent wavefunctions of the continuum-coupled Auger-dominated states. Past experiments by the ASACUSA collaboration [5, 6, 11] compared the calculated values with measured decay rates of the Auger-dominated states. The experimental decay rates of most Auger-dominated states were found to agree with the theoretical values.

\subsection{Formation process of the $\overline{\mathrm{p}} \mathrm{He}^{+}$atom in the highly excited states}

When an antiproton collides with a helium atom at electronvolt-scale energies, the metastable $\overline{\mathrm{pHe}}{ }^{+}$ atom is formed in the process,

$$
\overline{\mathrm{p}}+\mathrm{He} \rightarrow \overline{\mathrm{p}} \mathrm{He}^{+}+\mathrm{e}^{-} .
$$

The metastable atoms then slowly cascade down to lower states via radiative decays $\Delta n=\Delta l=-1$ (which keeps the vibrational quantum number $v=n-l-1[1,2]$ constant) with microsecond-scale 


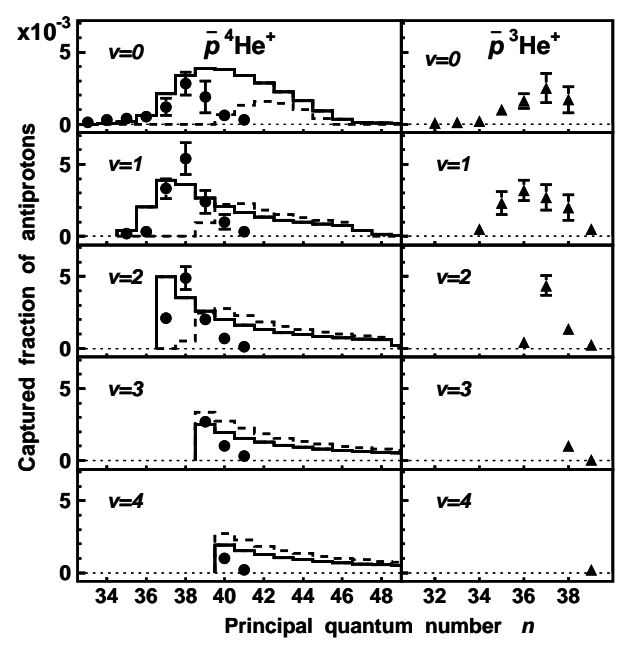

Figure 2. Measured distributions of primary populations in the $\overline{\mathrm{p}}^{4} \mathrm{He}^{+}$(filled circles with error bars) and $\overline{\mathrm{p}}^{3} \mathrm{He}^{+}$ (filled triangles) isotopes. Eq. (4) predicts the largest population at $n \sim 38$ for $\overline{\mathrm{p}}^{4} \mathrm{He}^{+}$, and $n \sim 37$ for $\overline{\mathrm{p}}^{3} \mathrm{He}^{+}$, which agreed with the experimental results. Theoretical $\overline{\mathrm{p}}^{4} \mathrm{He}^{+}$populations obtained from diabatic state [15] (solid lines) and semiclassical coupled-channel [16] (dashed lines) calculations are also shown. This figure is taken from Ref. [4].

lifetimes, and finally reach the Auger-dominated states (see figure 1). The ASACUSA collaboration has studied details of the formation process of the $\overline{\mathrm{p}} \mathrm{He}^{+}$atom $[4,14]$. This study is important to understand reaction dynamics of the Coulomb few-body system.

It has been believed that the antiproton is initially captured around the principal quantum number $n_{0}[12,13]$,

$$
n_{0}=\sqrt{M_{\overline{\mathrm{p}}}^{*} / m_{\mathrm{e}}}
$$

where $M_{\bar{p}}^{*}$ denotes the reduced mass of the $\overline{\mathrm{p}}$-He system. Eq. (4) was derived based on an assumption that the radius and the binding energy of the antiproton are same as those of the displaced $1 S$ electron. By laser spectroscopy of the $\overline{\mathrm{p}} \mathrm{He}^{+}$atom $[4,14]$, this hypothesis was experimentally verified. This can be seen in figure 2 , which shows measured distributions of primary populations (i.e., the number of antiprotons initially captured in a metastable state normalized to the total number of antiprotons stopped in the helium gas target).

The formation process in the highly excited states $n \geq 40$ has not been fully understood. The primary populations were theoretically calculated by several theorists [15-17] (two of the results are shown in figure 2). These theories predicted that the majority of antiprotons should be captured in the highly excited states. This prediction, however, deviated from the experiment in which very few populations were detected in those states (see figure 2). Additional information on this deviation may be obtained by the observation of the $1154.9 \mathrm{~nm}$ transition. We plan to deduce the primary populations and the decay rates in the highly excited metastable states.

\section{Nanosecond near-infrared laser}

A tunable laser with megawatt-scale output power at $\lambda=1154.9 \mathrm{~nm}$ was needed for this work. The requirement of the output power arose for the following reasons: a) The transition should be induced 
within a relatively short time $(\sim \mathrm{ns})$ compared to the lifetime of the metastable $\overline{\mathrm{pHe}} \mathrm{e}^{+}$atom, b) the signal of the antiproton annihilation induced by the laser should be strong enough for clear observation against the background resulting from spontaneous annihilations [10]. Taking the requirements into consideration, we have prepared a nanosecond near-infrared laser utilizing stimulated Raman scattering.

Raman scattering is a phenomenon in which the frequency $v_{\mathrm{p}}$ of an incident photon into a Raman gain medium is shifted to a lower frequency $v_{\mathrm{S}}=v_{\mathrm{p}}-v_{\mathrm{R}}$ (called the first Stokes shift), where the difference $v_{\mathrm{R}}$ is carried by a molecular vibration. We employed $\mathrm{H}_{2}$ gas as the Raman gain medium. $\mathrm{H}_{2}$ has an intense vibrational transition (called the $Q(1)$ transition) with the shift wavenumber $\tilde{v}_{R}=$ $\nu_{\mathrm{R}} / c=4155 \mathrm{~cm}^{-1}$. Thus, laser emission at $\lambda=1154.9 \mathrm{~nm}$ is possible by injecting a laser photon at $\lambda=780.4 \mathrm{~nm}$ into $\mathrm{H}_{2}$ gas. The conversion efficiency generally increases with longer pass lengths and gas pressures in a $\mathrm{H}_{2}$ cell, and higher intensities of the incident laser beam [18].

The near-infrared laser consisted of a nanosecond titanium sapphire (Ti:S) laser [19] and a 3-mlong Raman cell filled with $\mathrm{H}_{2}$ gas at room temperature and pressure of $600 \mathrm{kPa}$. The Ti:S laser produced a light pulse at $\lambda=780.4 \mathrm{~nm}$ with repetition rate of up to $1 \mathrm{~Hz}$, pulse length of $7 \mathrm{~ns}$, and pulse energy of $35 \mathrm{~mJ}$. This light pulse was shifted to $\lambda=1154.9 \mathrm{~nm}$ in the Raman cell. A nearinfrared pulse at $\lambda=1154.9 \mathrm{~nm}$ with pulse energy of $7 \mathrm{~mJ}$ was obtained. This energy is expected to be high enough for this work.

\section{Acknowledgements}

We would like to thank V. I. Korobov for theoretical calculations. This work was supported by the Grant-in-Aid for Specially Promoted Research (20002003) of MEXT, Japan, and the European Research Council (ERC-Stg).

\section{References}

[1] T. Yamazaki, N. Morita, R. S. Hayano, E. Widmann, and J. Eades, Phys. Rep. 366, 183 (2002).

[2] R. S. Hayano, M. Hori, D. Horváth, and E. Widmann, Rep. Prog. Phys. 70, 1995 (2007).

[3] M. Hori et al., Phys. Rev. Lett. 87, 093401 (2001).

[4] M. Hori et al., Phys. Rev. Lett. 89, 093401 (2002).

[5] H. Yamaguchi et al., Phys. Rev. A 66, 022504 (2002).

[6] H. Yamaguchi et al., Phys. Rev. A 70, 012501 (2004).

[7] M. Hori et al., Nature 475, 484 (2011).

[8] V. I. Korobov, Phys. Rev. A 77, 042506 (2008); private communication, (2012).

[9] A. Sótér, D. Barna, and M. Hori (in preparation).

[10] M. Hori et al., Nucl. Instrum. Methods Phys. Res. A 496, 102 (2003).

[11] M. Hori et al., Phys. Rev. A 57, 1698 (1998); 58, 1612(E) (1998).

[12] E. Fermi and E. Teller, Phys. Rev. 72, 399 (1947).

[13] A. S. Wightman, Phys. Rev. 77, 521 (1950).

[14] M. Hori et al., Phys. Rev. A 70, 012504 (2004).

[15] K. Ohtsuki, unpublished theoretical data, (1992).

[16] G. Ya. Korenman, Hyperfine Interact. 101 - 102, 81 (1996); Nucl. Phys. A692, 145c (2001).

[17] W. A. Beck, L. Wilets, and M. A. Alberg, Phys. Rev. A 48, 2779 (1993).

[18] N. Bloembergen, Am. J. Phys. 35, 989 (1967).

[19] M. Hori and A. Dax, Opt. Lett. 34, 1273 (2009). 